HEFAT2014

$10^{\text {th }}$ International Conference on Heat Transfer, Fluid Mechanics and Thermodynamics

14 - 16 July 2014

Orlando, Florida

\title{
INTERNAL FLOW PATTERNS OF AN EVAPORATING MULTICOMPONENT DROPLET ON A FLAT SURFACE
}

\author{
M.H. HE, H.H. Qiu* \\ *Author for correspondence \\ Department of Mechanical and Aerospace Engineering, \\ The Hong Kong University of Science and Technology, \\ Clear Water Bay, Kowloon, Hong Kong SAR, China \\ E-mail: meqiu@ust.hk
}

\begin{abstract}
Evaporation processes of droplets with different ethanol/water volume ratios $(0 \%$ ethanol $/ 100 \%$ water, $5 \%$ ethanol $/ 95 \%$ water and $10 \%$ ethanol $/ 90 \%$ water, respectively) on a hydrophobic silicon dioxide surface covered by a layer of FAS were investigated by PIV method. It was found that the evaporation process of heated two-component droplets can be divided into three stages, downward vortex stage, transition stage and upward vortex stage sequentially, while for a pure water droplet only the upward vortex stage exists. The mechanism of those three stages was analysed in detail, combining previous theories of Marangoni effects, buoyancy effect and experiment results. Besides, it was also found that initial composition and input power affected the duration of the three stages, especially the downward vortices and transition stage which are related to ethanol evaporation: In low input power, the transition stage of the high ethanol fraction droplet occurred earlier and lasted a shorter time than the low ethanol fraction droplet, while, in high input power, the transition stage of the low ethanol fraction droplet occurred earlier and lasted a shorter time than the high ethanol fraction droplet. That phenomenon was also analysed with ethanol evaporation in different thermal and solutal conditions.
\end{abstract}

\section{INTRODUCTION}

The evaporation of droplets is important in many applications such as coatings (automobile exteriors and photoresist deposition), droplet-based microfluidics [10], inkjet printing [9], film formation [2] and even spotting DNA microarray data [1]. Evaporation of monocomponent droplets has been studied for decades [11-15]. Recently, experimental and numerical investigations have been focused on multicomponent evaporation because it has more applications and its mechanism is more complicated than monocomponent droplets [2-7, 16-18]. Among the first researchers to study multicomponent droplets, Newbold and Amundson [16] derived an accurate model to describe the evaporation of a multicomponent droplet near its boiling point. Tamim and Hallett [17] present a model for the evaporation of droplets of multicomponent liquids in which the mixture composition, properties and vapor-liquid equilibrium are described by the continuous thermodynamics methods. In another aspect of experiments, Zhao and Qiu [18] used rainbow refractometer and PDA method to measure temperature gradient within an evaporating microdroplet. Kang and Lee [19] first used PIV to demonstrate the existence of internal flow in sessile ethanol/water droplets which was conjectured to be driven by uneven evaporation of species in a multicomponent mixture, but they did not further analyze the mechanism. It was also predicted that thermal and solutal Marangoni effects $[5,7]$ will generate internal convection based on linear Marangoni stability modeling. Later, Mandal and Bakshi [6] proved this prediction by reporting evidence that volatile compositions introduce internal convection in a suspended multicomponent droplet while evaporating in an atmospheric environment.

However, the question that remains is: how can a volatile composition influence the internal flow pattern of the whole evaporation process as it evaporates faster. Besides, few researchers investigated the evaporation of ethanol/water droplets on a heated surface. In the present work, internal flow patterns of evaporating multicomponent droplets were investigated systematically using the PIV method. It was found the internal flow patterns of multicomponent droplets can be divided into three stages which were significantly different to monocomponent droplets. The mechanisms of each stage were analyzed combining previous theories of thermal and solutal Marangoni effects. It was also found that ethanol concentration and input power had great influence on the duration of the three stages, which was studied in relation to the ethanol evaporation rate in different conditions.

\section{EXPERIMENTAL SETUP}

Figure 1 shows the experimental schematics for droplet evaporation experiments. Droplets with a constant volume of 6 
$\mu \mathrm{l}$ (diameter about $2.5 \mathrm{~mm}$ when suspended) will be generated by the micro syringe (Hamilton, $10 \mu 1700$ series hand fitted MICROLITER syringe) and gently deposited on the hydrophobic ITO heater surface. Each droplet was deposited in the same place to ensure reproducibility. The liquid for the experiments was deionized water based mixtures with ethanol, in which the ethanol concentration was varied to produce volume ratios of $0 \%, 5 \%$ and $10 \%$.

The substrate of $2 \mathrm{~cm} \times 2 \mathrm{~cm}$ was made of transparent silicon dioxide coated with an ITO layer of 100nm thickness, which acted as a resistive heater, with its surface chemically modified with a fluoroalkylsilane $\left(\mathrm{C}_{6} \mathrm{~F}_{13}\left(\mathrm{CH}_{2}\right)_{2} \mathrm{Si}\left(\mathrm{OCH}_{3}\right)_{3}\right)$ monolayer to make it hydrophobic. The initial contact angles for $0 \%, 5 \%$ and $10 \%$ ethanol volume ratio are 115,109 , and 97 degree on the modified surface, respectively. To study droplet evaporation under different thermal conditions, the input power to the heater varied from $0.25 \mathrm{~W}$ to $2.5 \mathrm{~W}$.

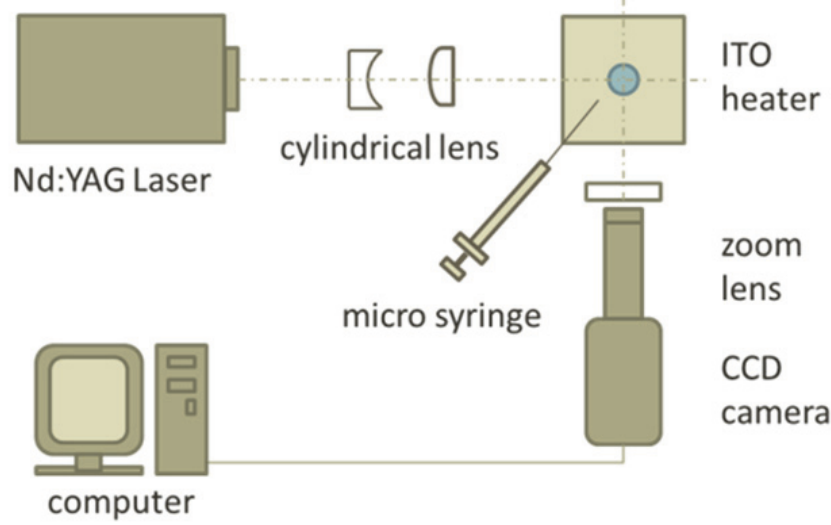

Figure 1 Schematics of droplet evaporation experiments

To apply the PIV method for internal flow investigation, seeding particles (Fluoro-Max ${ }^{\mathrm{TM}}$ R0300, Thermo Scientific Lmt.), having a mean diameter of $3.2 \mu \mathrm{m}$ and a density of 1.05 $\mathrm{g} / \mathrm{cm} 3$, were used for revealing internal flow during droplet evaporation. In this study, the concentration of seeding particles was $0.05 \%$ in volume ratio and the number density was $2.91 \mathrm{E} 7$ per ml. An ion laser was used for illumination. After passing through a concave cylindrical lens and a convex cylindrical lens, the laser beam was expanded to a laser light sheet with a thickness about $0.3 \mathrm{~mm}$, which was much smaller than the diameter of the droplet. A CCD camera (MotionXtra HG-100K, Redlake lmt.), applied to record images of profile changing and flow pattern inside the droplet, was attached with a long distance zoom lens (Zoom 6000, Navitar Inc.), with the axis of the lens perpendicular to the laser beam. According to the specification of the zoom lens, the depth of focus ranged from $0.24 \mathrm{~mm}$ at the lowest magnification to $0.02 \mathrm{~mm}$ at the highest magnification, which is smaller than the laser sheet. The local surroundings were kept at $296 \mathrm{~K}$ and $50 \% \mathrm{RH}$. Each experiment for droplet evaporation was carried out more than three times to identify the reproducibility in time variations.
According to the recorded images, the reproducibility of the internal flow pattern was identified.

\section{RESULTS AND DISCUSSION}

In order to better study the influence of the second component, ethanol in this study, to the evaporation process, it was necessary to study the evaporation process of pure water as reference.

Once the droplet was gently deposited on the flat surface, the internal flow pattern of two vortices appeared, which were symmetric and of upward direction. Figure 2 shows the typical internal flows of pure water droplets evaporating on the ITO heater surface with input power of $0.5 \mathrm{~W}, 1 \mathrm{~W}$ and $2 \mathrm{~W}$, respectively. Those internal flow patterns were caused by the input heat flux. When the input power is high, temperature inside the droplet was non-uniform: liquid near apex of droplet and air-liquid interface is cool and liquid near heater surface is hot, especially near the centre of the droplet base. This temperature gradient leads to surface tension and density gradient inside the droplet, both of which result in buoyancy induced convection and make the water go up, leading to symmetric upward vortices. As the input power increased, the temperature gradient became larger and led to a higher internal flow rate, in agreement with Figure 2. The average internal flow rates calculated from raw images were $0.13 \mathrm{~mm} / \mathrm{s}, 0.44$ $\mathrm{mm} / \mathrm{s}$ and $0.77 \mathrm{~mm} / \mathrm{s}$ for input power of $0.5 \mathrm{~W}, 1 \mathrm{~W}$ and $2 \mathrm{~W}$ respectively. Evaporation rate was also enhanced with the increased input power because shear stress produced by internal flow constantly entrains surrounding gas into the region near the interface and this motion constantly removes evaporated vapour from the interface and brings in fresh air to the droplet surface, increasing the heat convection [8]. It is worth mentioning that only internal flow patterns of upward vortices appeared.

However, evaporation of multicomponent ethanol/water droplets was significantly different to pure droplets. First of all, the evaporation duration of multicomponent droplets was shorter than pure water droplets because ethanol has a higher vapour pressure and lower boiling point than water. Figure 3 shows droplet evaporation duration of droplets with different composition and input power. It is apparent that under same thermal condition, droplets with a higher ethanol concentration evaporated faster. Besides, it was also found that evaporation enhancement compared to pure water droplets of multicomponent droplets was undermined when the input power was high, as shown in Figure 4, which can be caused by fast evaporation of ethanol under high input power. However, to have a better understanding of the mechanism of multicomponent droplet evaporation, it was necessary to investigate the internal flow patterns of the whole evaporation process.

In fact, the internal flow patterns of ethanol-DI water mixture droplets were far different from pure water droplets. While the internal flow of pure water droplets had only one upward vortex stage, the ethanol/water mixtures droplets had three stages (internal flow patterns) during evaporation: downward vortex stage (Figures 5 and 6), transition stage (Figures 7 and 8) and upward vortex stage (Figures 9 and 10), 
respectively. Until now, these three flow patterns of multicomponent droplet evaporation had not been reported.

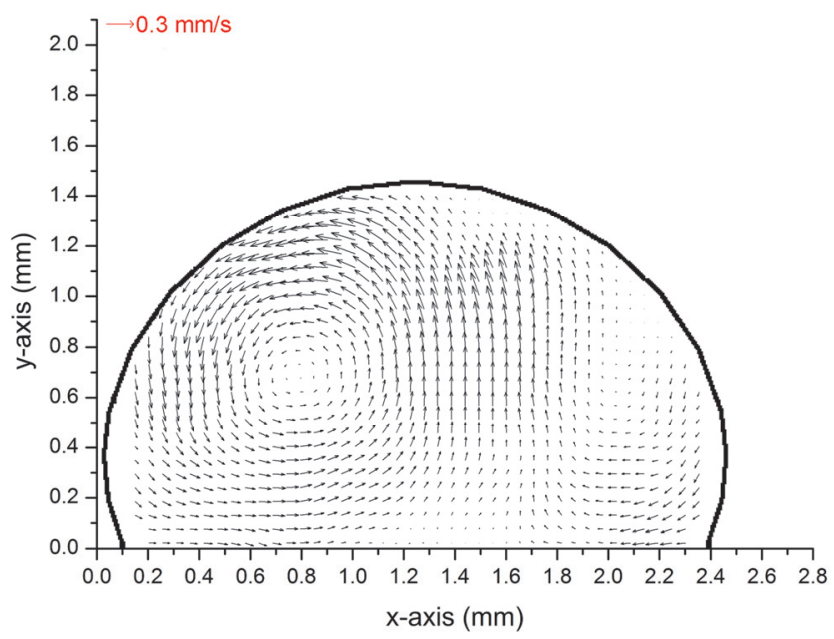

(a) Input power $0.5 \mathrm{~W}$

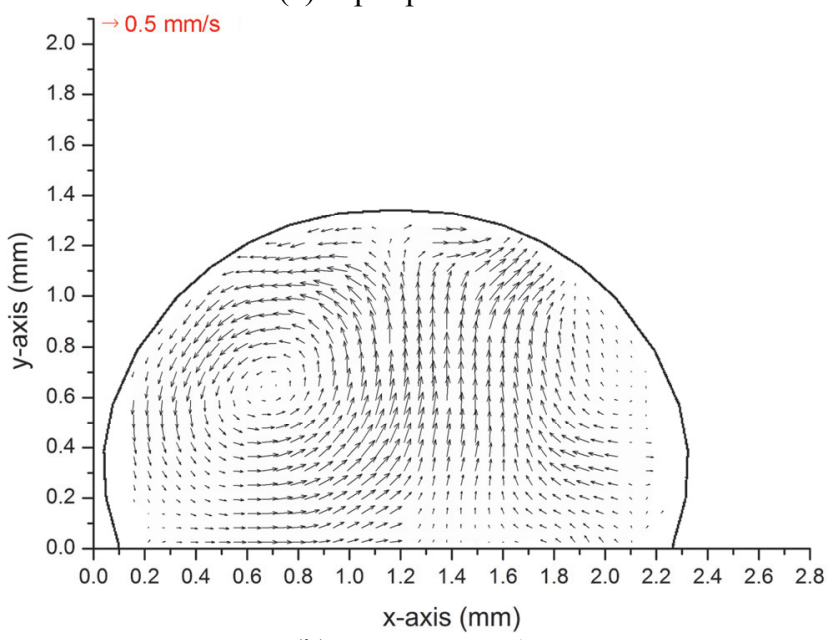

(b) Input power $1 \mathrm{~W}$

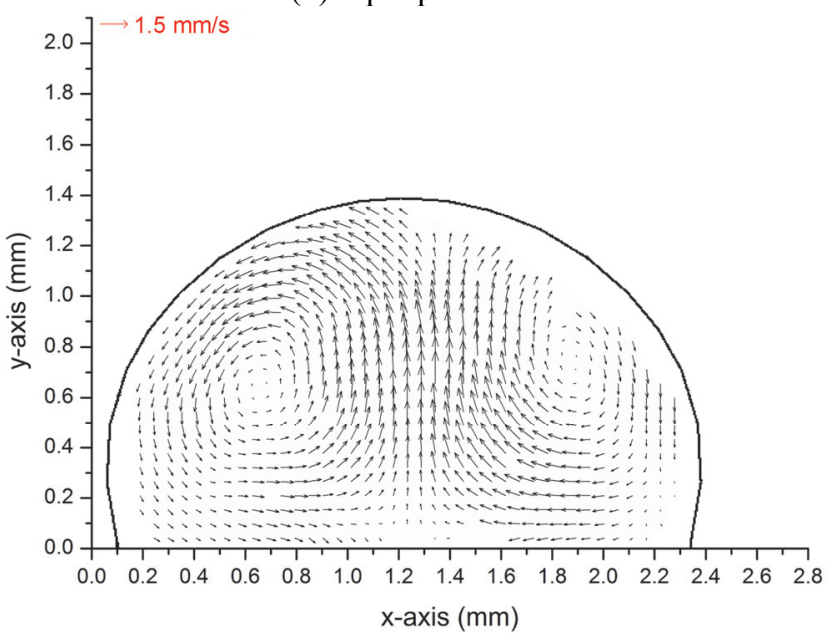

(c) Input power $2 \mathrm{~W}$

Figure 2 Internal flow pattern (upward vortices) of pure water droplet under different input power: (a) $0.5 \mathrm{~W}$, (b) $1 \mathrm{~W}$ and (c) $2 \mathrm{~W}$.

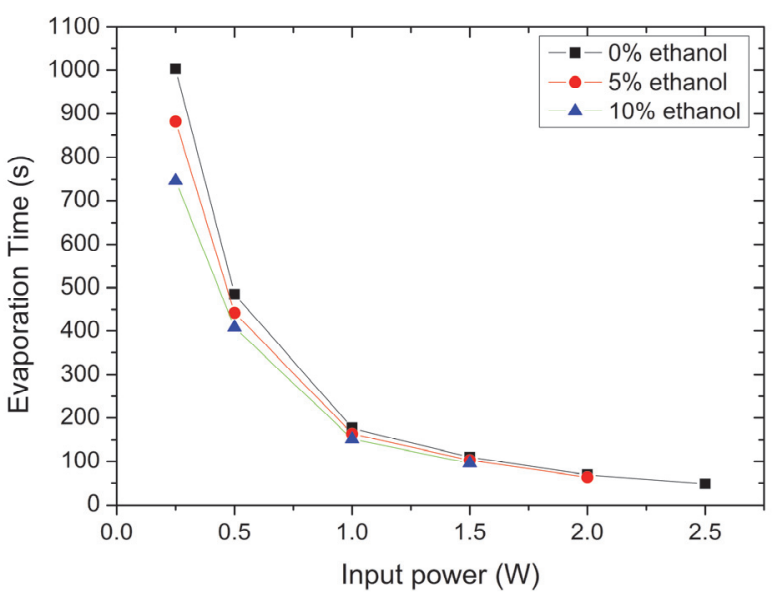

Figure 3 Evaporation duration of droplets with different ethanol/water volume fraction $(0 \%, 5 \%$ and $10 \%)$ under different thermal condition (input power from $0.25 \mathrm{~W}$ to $2.5 \mathrm{~W}$ ).

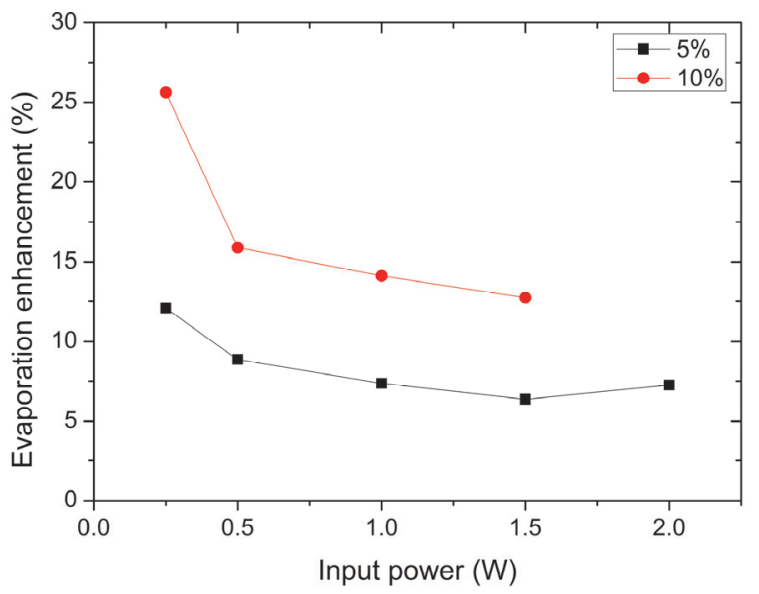

Figure 4 Evaporation enhancement of droplet ethanol/water volume fraction $(0 \%, 5 \%$ and $10 \%)$ under thermal condition (input power from $0.25 \mathrm{~W}$ to $2.5 \mathrm{~W}$ )

Once the droplet was gently deposited on the heater surface, the flow pattern of downward vortices appeared immediately and lasted the rest of evaporation process. Figures 5 and 6 show the typical internal flow patterns and internal flow rate of the downward vortex stage of multicomponent droplets (ethanol volume fraction of $5 \%$ and $10 \%)$ with low input power $(0.25 \mathrm{~W})$ and high input power $(1 \mathrm{~W})$. During the downward vortex stage, the liquid flowed from the apex to the base of the droplet at a very high velocity and formed two symmetric vortices thus called the downward vortices stage. It is apparent that ethanol in the mixture droplet will evaporate first because the ethanol has a lower boiling point and latent heat. Therefore, once the droplet contacted the heater surface, the volatile composition (ethanol) evaporated preferentially, especially near the contact line where the evaporation mass flux was largest, and the ethanol's concentration of droplet rapidly decreased. As strong evaporation occurred near the contact line during this stage, the 
ethanol concentration near the contact line will be low compared to the droplet's centre, which led to large surface tension gradient and resulted in Marangoni convection flowing from the centre of the droplet to the contact line, forming downward vortices. These symmetric and significant vortices were also reported in droplets of pure alcohols like methanol and ethanol by [8], which confirmed that the solutal effect of ethanol was mainly driving the evaporation in this stage and generated internal flow patterns of symmetric downward vortices.

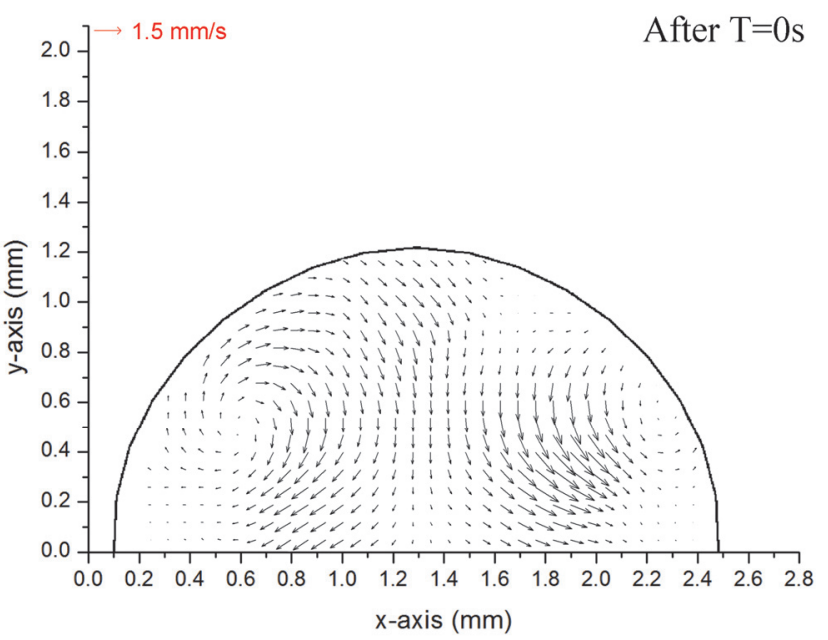

(a) $5 \%$ ethanol volume fraction, $0.25 \mathrm{~W}$ input power

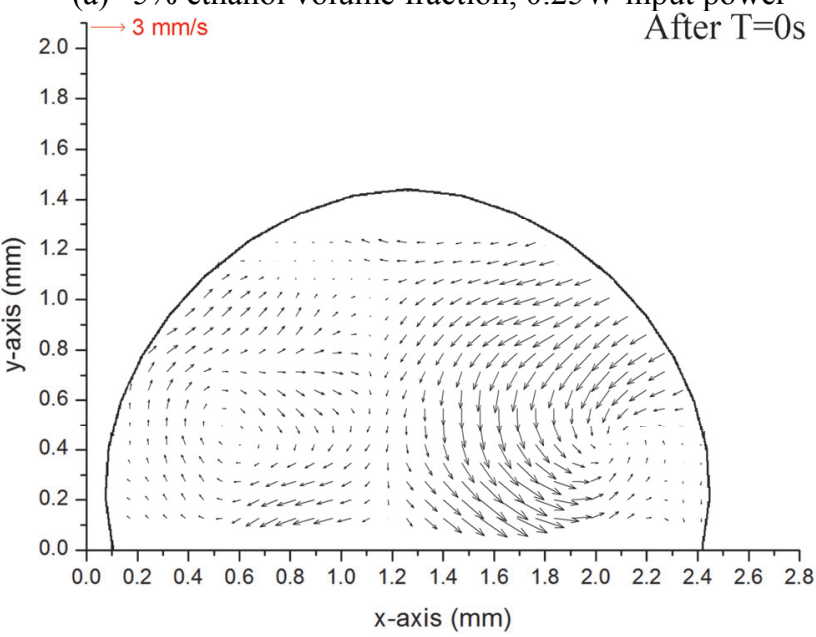

(b) $5 \%$ ethanol volume fraction, $1 \mathrm{~W}$ input power

Figure 5 Internal flow patterns of the downward vortices stage of multicomponent droplets (ethanol volume fraction of $5 \%$ ) with low input power $(0.25 \mathrm{~W})$ and high input power $(1 \mathrm{~W})$

Figures 5 and 6 also show that the internal flow rate of this stage increased while the input power increased: for droplets of $5 \%$ ethanol volume fraction, the average internal flow rates calculated from raw images were $0.69 \mathrm{~mm} / \mathrm{s}$ for $0.25 \mathrm{~W}$ input power and $1.12 \mathrm{~mm} / \mathrm{s}$ for $1 \mathrm{~W}$ input power, while for droplets of $10 \%$ ethanol volume fraction the average internal flow rates were $1.01 \mathrm{~mm} / \mathrm{s}$ for $0.25 \mathrm{~W}$ and $1.35 \mathrm{~mm} / \mathrm{s}$ for $1 \mathrm{~W}$. Compared to evaporating pure water droplets under the thermal condition, the internal flow rate of multicomponent droplets was significantly larger, which also implied that the evaporation of ethanol was driving the evaporation in the downward vortices stage.

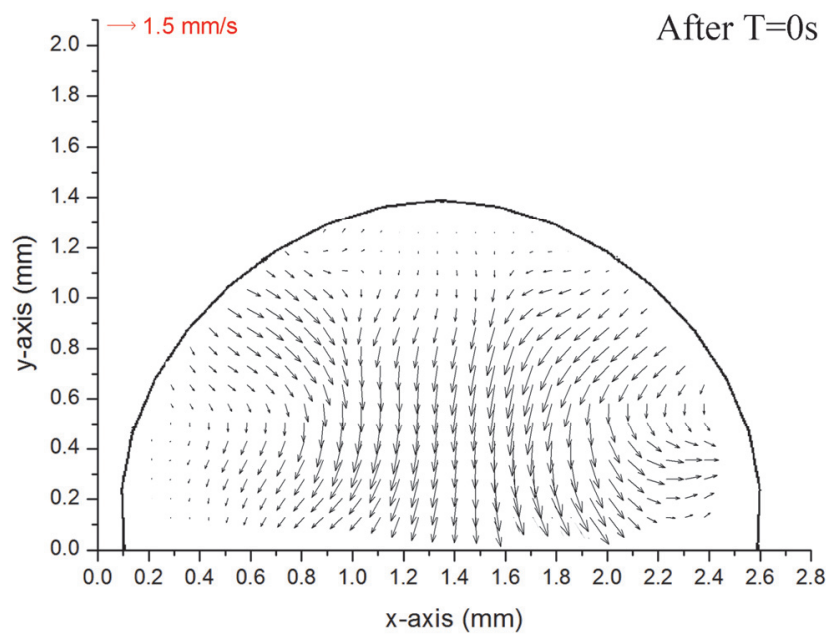

(a) $10 \%$ ethanol volume fraction, $0.25 \mathrm{~W}$ input power

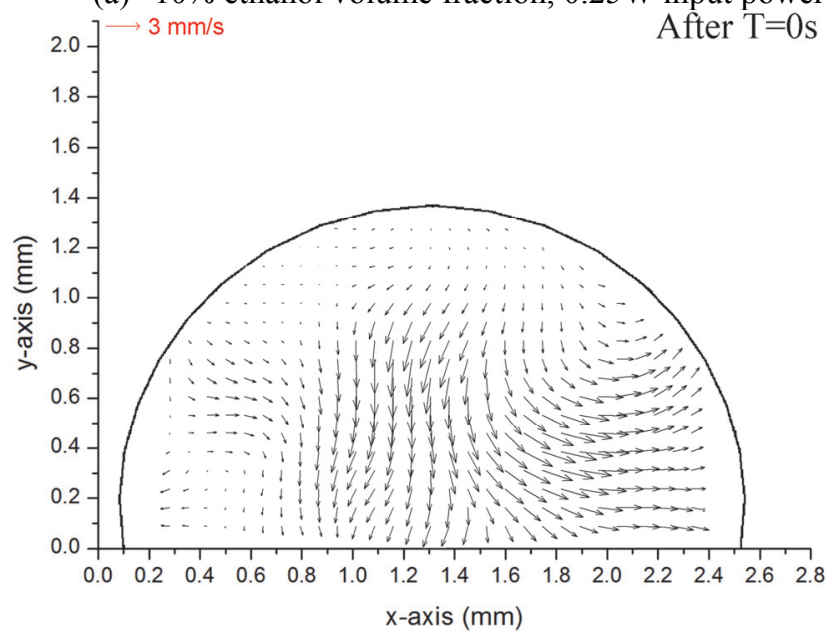

(b) $10 \%$ ethanol volume fraction, $1 \mathrm{~W}$ input power

Figure 6 Internal flow patterns of the downward vortices stage of multicomponent droplets (ethanol volume fraction of $10 \%$ ) with low input power $(0.25 \mathrm{~W})$ and high input power $(1 \mathrm{~W})$

After the downward vortices stage, the transition stage occurred, in which the internal flow was rather unstable. During most of the time in this stage, only one vortex appeared inside the droplet but did not stay at the same place and always "ran" along the air-liquid interface. Sometimes the vortex appeared in the left side of the droplet and sometimes it appeared in the right side, as shown Figures 7 and 8. However, it is worth mentioning that in this stage the vortex always went from the apex to the base of the droplet, quite similar to the direction of the downward vortices stage, which implied the ethanol evaporation still affected the evaporation of multicomponent droplets in the transition stage

As the first stage continued, the concentration of ethanol decreased and evaporation of ethanol decayed, which implied 
that the solutal Marangoni effect was not the main driving force but both thermal Marangoni and buoyancy effect should be considered. At the beginning of transition stage, when the droplet was not highly heated up as ethanol evaporation in the downward stage released large heat, the buoyancy effect was not significant and the thermal Marangoni effect acted as major opposite role to solutal Marangoni effect.

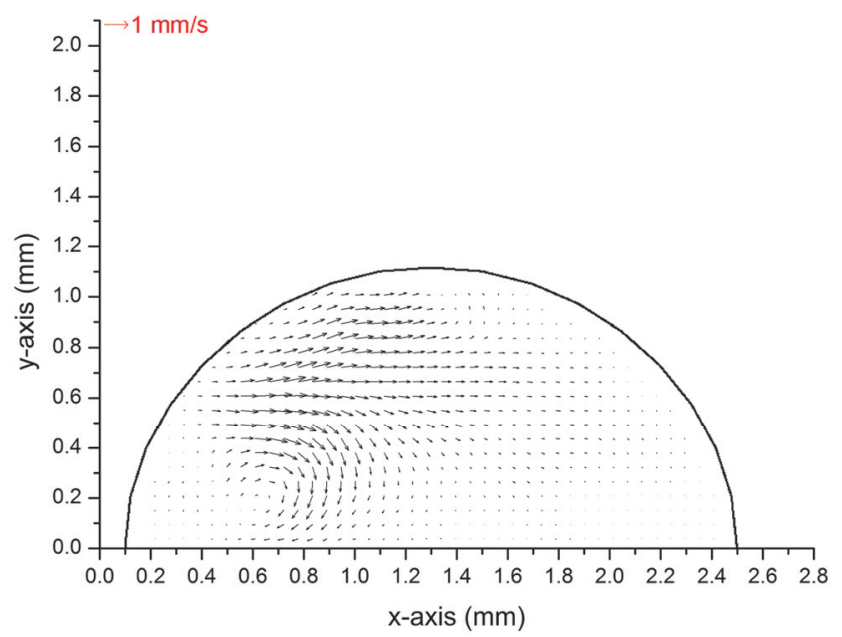

(a) Left vortex in transition stage

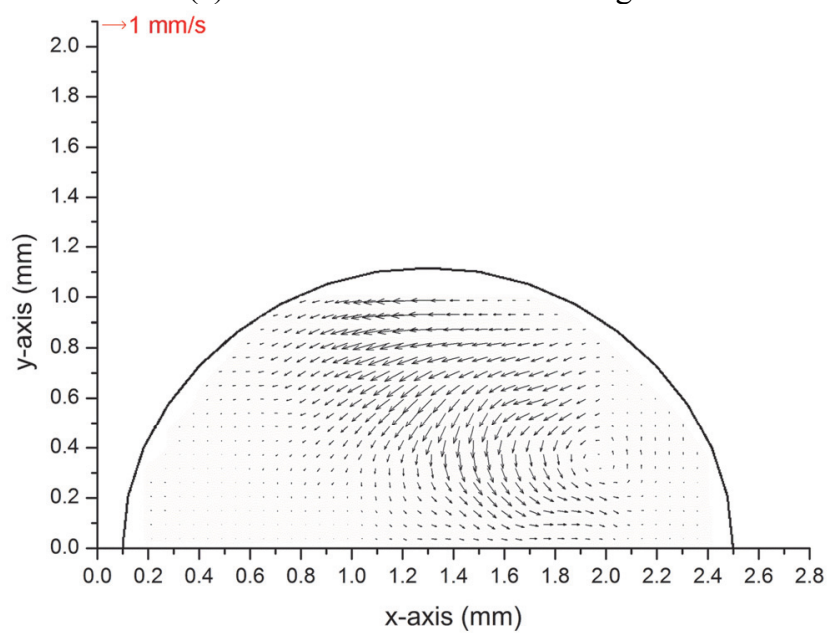

(b) Right vortex in transition stage

Figure 7 Internal flow patterns of the transition stage of 5\% ethanol volume fraction with input power of $0.25 \mathrm{~W}$ after $\mathrm{T}=175 \mathrm{~s}$.

For a system composed of water-ethanol, which has a negative value of rate of change of surface tension with temperature and concentration of the volatile component, the thermal and solutal Marangoni affects oppose each other [7]. Those opposite effects have significant influence near the surface and thus induce Marangoni instability and unstable oscillatory convection inside the droplet, as reported in [6], in which droplets for experiments had similar diameter $(2.7 \mathrm{~mm})$ to ours.

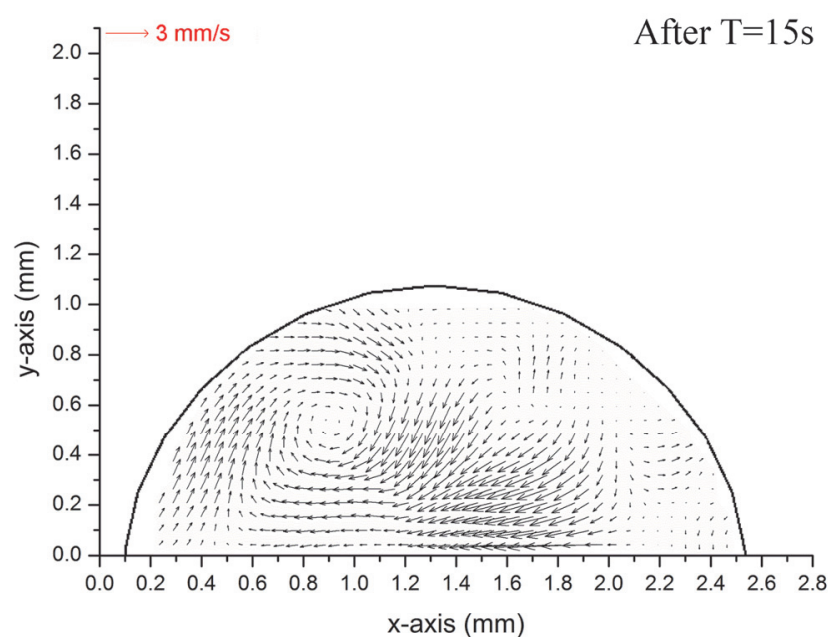

(a) 5\% ethanol volume fraction, $1 \mathrm{~W}$ input power

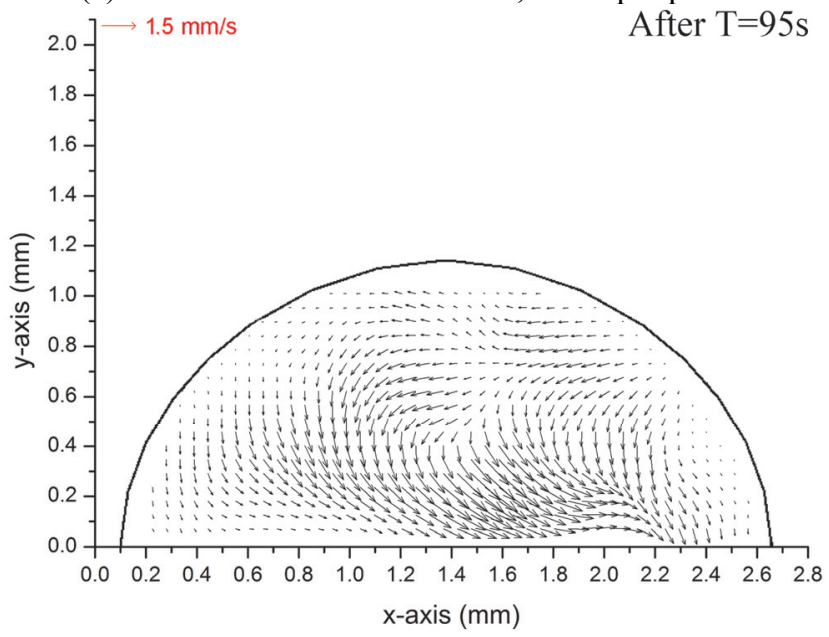

(b) $10 \%$ ethanol volume fraction, $0.25 \mathrm{~W}$ input power

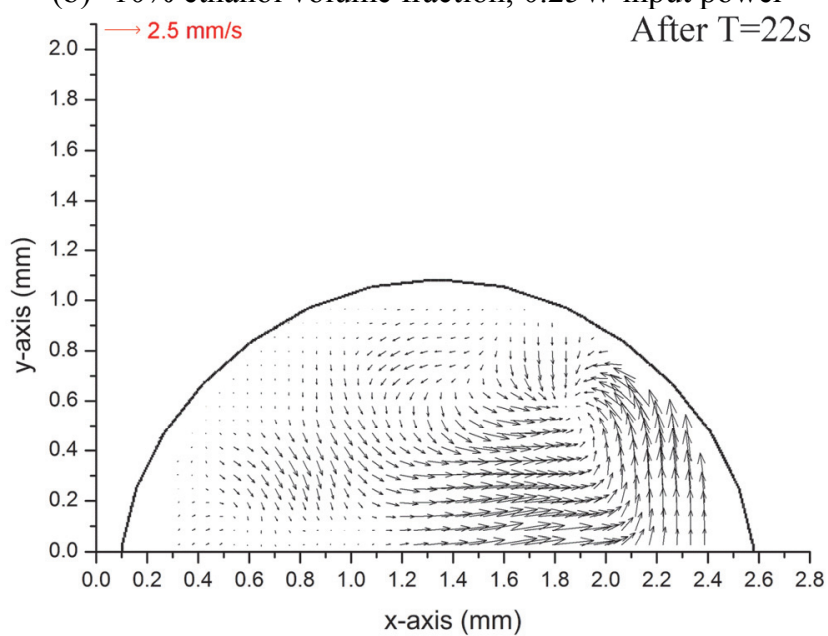

(c) $10 \%$ ethanol volume fraction, $1 \mathrm{~W}$ input power

Figure 8 Internal flow patterns of the transition stage of multicomponent droplets (ethanol volume fraction of $5 \%$ and $10 \%)$ with low input power $(0.25 \mathrm{~W})$ and high input power

(1W). 
As heat was supplied from the bottom, ethanol concentration near contact line was relatively low, the same as downward vortices stage. Capillary stresses (Marangoni stresses) will pull the liquid from the region of lower surface tension, where ethanol concentration is high, to a region with higher surface tension where ethanol concentration is lower.

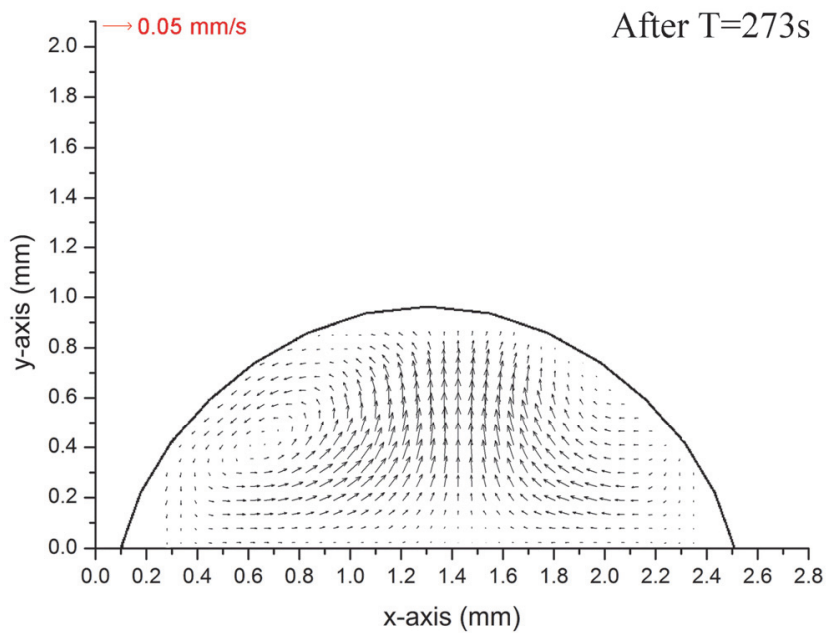

(a) $5 \%$ ethanol volume fraction, $0.25 \mathrm{~W}$ input power

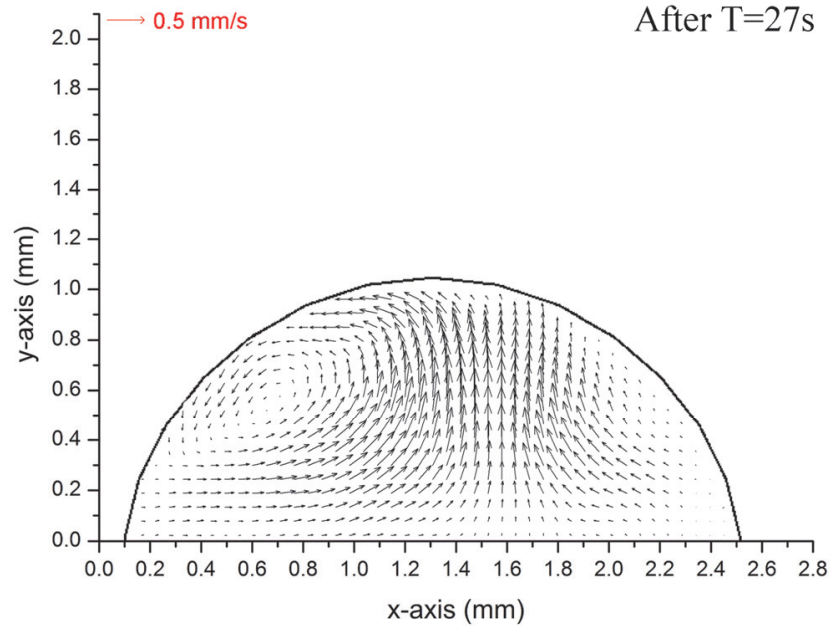

(b) $5 \%$ ethanol volume fraction, $1 \mathrm{~W}$ input power

Figure 9 Internal flow patterns of the upward vortices stage of multicomponent droplets (ethanol volume fraction of 5\%) with low input power $(0.25 \mathrm{~W})$ and high input power $(1 \mathrm{~W})$

To satisfy mass conservation, liquid will be converted from the droplet centre to the region where ethanol concentration was increased by perturbation, which will maintain the locallyenriched ethanol concentration by transport of ethanol-rich mixture to the surface near contact line. Thus solutal effects will sustain this flow, causing higher non-uniform distribution of ethanol at the surface, which will be a destabilizing factor in this case. This explained why convection always flowed from the center to the contact line, as shown in Figures 7 and 8.Meanwhile, as flow from the droplet inside to the surface locally increases the ethanol concentration at certain locations. The surface at these locations will cool down quickly because the higher ethanol concentration the faster evaporation and be cooler than other surface locations where there is less ethanol. The surface temperature gradient generated by this flow will induce thermal surface tension gradients that will act opposition to the solutal which means thermal effects will be a stabilizing factor for a water-ethanol mixture, which made the vortex unstable and slow down.

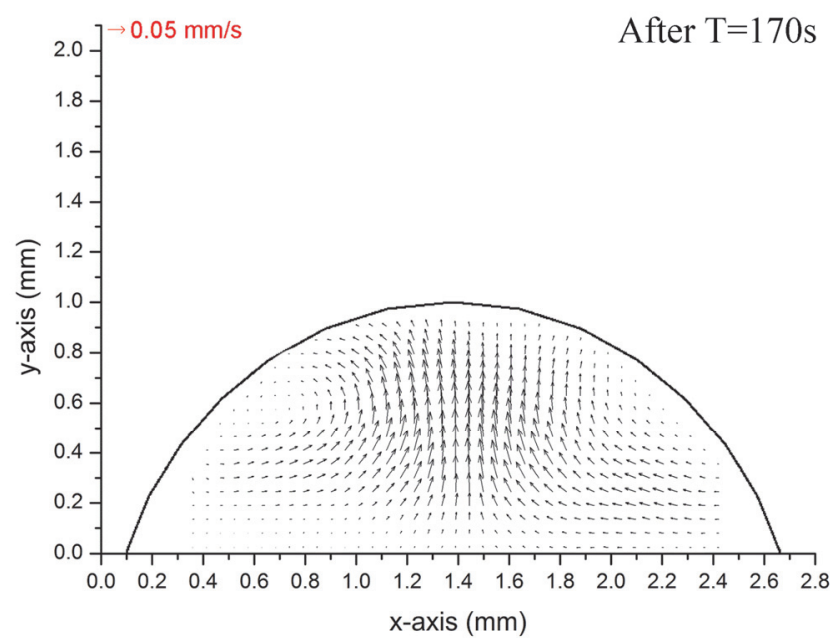

(a) $10 \%$ ethanol volume fraction, $0.25 \mathrm{~W}$ input power

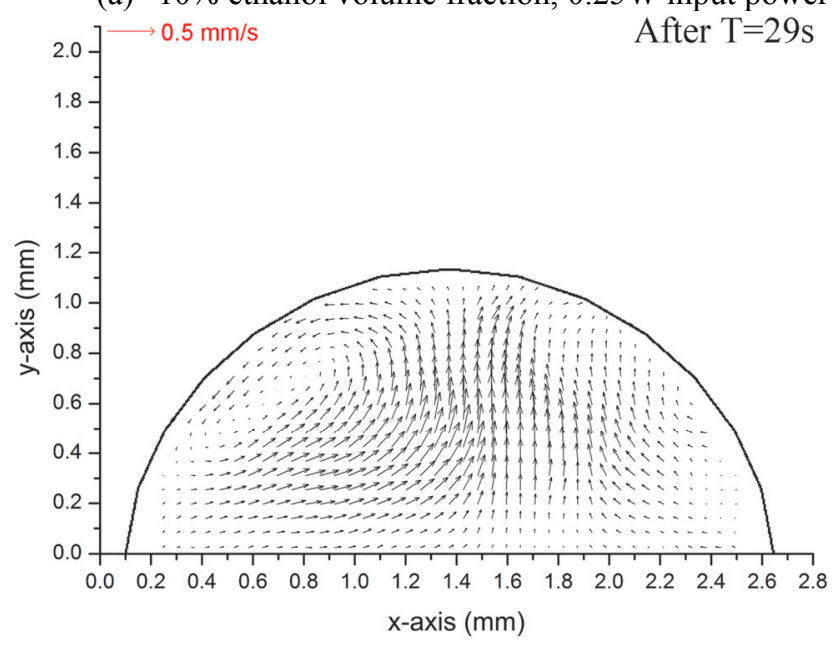

(b) $10 \%$ ethanol volume fraction, $0.25 \mathrm{~W}$ input power

Figure 10 Internal flow patterns of the upward vortices stage of multicomponent droplets (ethanol volume fraction of $10 \%$ ) with low input power $(0.25 \mathrm{~W})$ and high input power $(1 \mathrm{~W})$

However, as ethanol concentration continuously decreased and the droplet was further heated, the stabilizing effect of thermal Marangoni effect was undermined because temperature of droplet center was high and temperature gradient generated by internal flow was reduced (even contrary), and buoyancy effect acted as a major opposite role. While solutal Marangoni effect produced vortex flowing from center to contact line, buoyancy induced convection generated vortices flowing inversely, from contact line to center. It is because surface tension and density gradient resulted from temperature gradient 
between bottom and apex of the droplet. As both thermal Marangoni effect and buoyancy played inverse roles to solutal Marangoni effect, this flow slowed and stopped as the ethanol concentration decreased in this location which meant less solutal effect. Then in a new, local-rich ethanol location, this kind of flow repeated until most of the ethanol eventually gasified. After this stage, the evaporation was suddenly (within 2 seconds) changed to mainly buoyancy induced convection driven, when the upward vortices stage began, at which stage the internal flow rate was slowest, as shown Figures 9 and 10. It can be mentioned that the pure water droplet only exhibited the upward vortices stage (Figure 2), which proved that most of the ethanol had already gasified from the heated multicomponent droplet when the transition stage ended. As shown in Figures 7 and 8, the internal flow rate increased with increased input power and increased ethanol concentration, the same as the downward vortices stage: for droplets of $5 \%$ ethanol volume fraction, the average internal flow rates calculated from raw images were $0.38 \mathrm{~mm} / \mathrm{s}$ for $0.25 \mathrm{~W}$ input power and $0.92 \mathrm{~mm} / \mathrm{s}$ for $1 \mathrm{~W}$ input power, while for droplets of $10 \%$ ethanol volume fraction the average internal flow rates were $0.59 \mathrm{~mm} / \mathrm{s}$ for $0.25 \mathrm{~W}$ and $1.01 \mathrm{~mm} / \mathrm{s}$ for $1 \mathrm{~W}$. The evaporation rate was also enhanced for high internal flow rate.

As both the downward vortices stage and transition stage related to ethanol evaporation and the end of the transition stage indicated ethanol evaporation completed, it is worth investigating the influence of ethanol concentration and input power on ethanol evaporation and the whole evaporation of multicomponent droplet. Considering the duration of ethanol evaporation (including the downward vortices and transition stage), interesting phenomenon was found. Seen in Figure 11, with low input power, the downward vortices stage and transition stage of the high ethanol fraction droplet lasted a shorter time than the low ethanol fraction droplet, while, with high input power, both stages of high ethanol fraction droplet lasted longer. This phenomenon also implied that, for low input power, ethanol in high ethanol concentration droplets evaporated faster, but with high input power, ethanol in low ethanol droplets evaporated faster. The average internal flow rate also confirmed this assumption. With low input power, the average internal flow rate of the downward vortices stage (1.01 $\mathrm{mm} / \mathrm{s})$ and transition stage $(0.59 \mathrm{~mm} / \mathrm{s})$ for $10 \%$ volume fraction was $46 \%$ and $55 \%$ faster than $5 \%$ volume fraction; with high input power, the average internal flow rate of the downward vortices stage $(1.35 \mathrm{~mm} / \mathrm{s})$ and transition stage $(1.01$ $\mathrm{mm} / \mathrm{s}$ ) for $\%$ volume fraction was only $17 \%$ and $10 \%$ faster than $5 \%$ volume fraction. These comparisons indicate that the internal flow rates for droplets of 5\% and $10 \%$ ethanol volume fraction were close when the input power was large.

When the input power was low, the reason for the short duration of the upward vortices and transition stage for high ethanol fraction was the large entropy of mixing when the ethanol fraction was higher, and the large gradient of ethanol concentration between air/liquid interface, leading to the large driving force and highly unsteady internal flow, increased the ethanol evaporating rate. However, when the input power was high, air near the contact line was easily saturated because ethanol evaporated fast near the base line in the very beginning, compared with low input power. If air near the droplet was saturated, the concentration gradient between liquid and air was small and the gas entrained into the interface by internal flow was no longer 'fresh', acted as a barrier preventing ethanol molecules transporting into air. It implied that the effect of the concentration gradient between liquid and air was small and the evaporation was mainly driven by heat flux from the heater with high input power. Thus, for high ethanol concentration droplets with high input power, the larger amount of ethanol, the longer time it takes to evaporate with the same input heat flux.

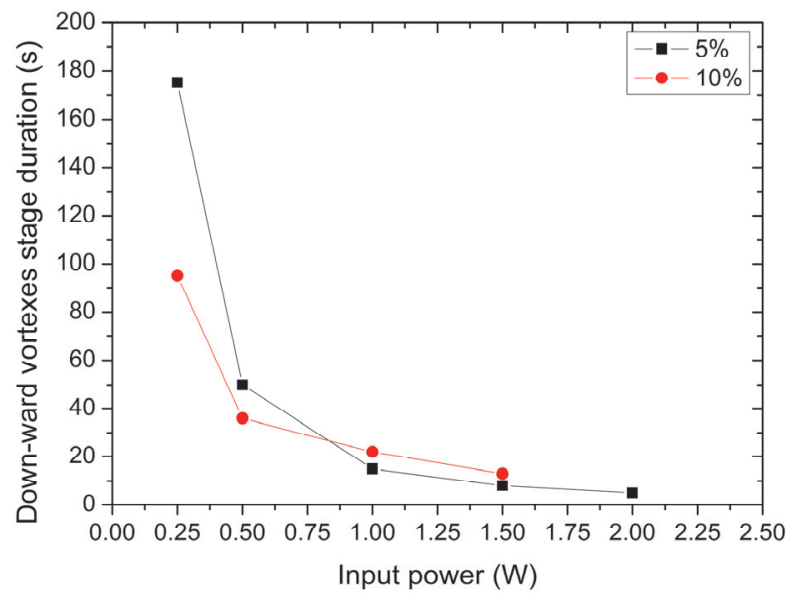

(a) Downward vortices stage duration of multicomponent droplets (ethanol volume fraction of $5 \%$ and $10 \%$ ) from low input power $(0.25 \mathrm{~W})$ to high input power $(1 \mathrm{~W})$

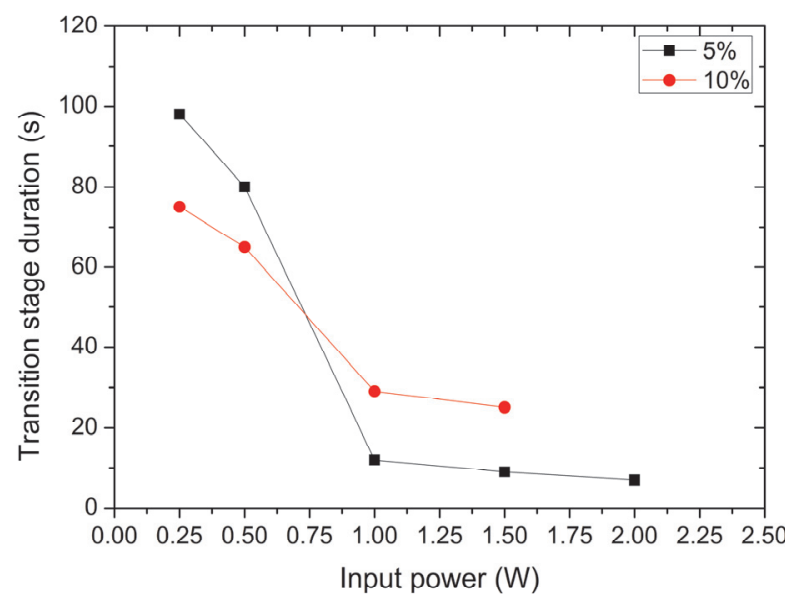

(b) Transition stage duration of multicomponent droplets (ethanol volume fraction of $5 \%$ and $10 \%$ ) from low input power $(0.25 \mathrm{~W})$ to high input power $(1 \mathrm{~W})$

Figure 11 Duration of down-ward vortices and transition stage duration of multicomponent droplets (ethanol volume fraction of $5 \%$ and $10 \%$ ) form low input power $(0.25 \mathrm{~W})$ to high input power $(1 \mathrm{~W})$ 


\section{CONCLUSION}

The present work and results show that the evaporation process and flow patterns of ethanol-water mixture droplets are much different from pure water droplets when heated. Multicomponent droplets have three stages of evaporation (downward vortices stage, transition stage and upward vortices stage, sequentially), while only the upward vortices stage is present for pure water. Among the three stages, the downward vortices stage and transition stage were introduced by evaporation of ethanol and the end of the transition stage indicated the complete evaporation of ethanol. As the evaporation of ethanol was much faster than water, the internal flow rates of these two stages were much higher than the upward vortices stage. Besides, the high internal flow rate of these two stages enhanced the evaporation because the shear stress produced by the fast internal flow enhanced the heat transfer and material transportation, which was confirmed by the experiment results.

Also, the composition of droplet and thermal condition had a significant influence on duration of the three internal flow patterns. It is interesting to find that, with low input power, the downward vortices stage and transition stage of high ethanol fraction droplets lasted a shorter time than the low ethanol fraction droplets, while both stages of high ethanol fraction droplets lasted longer with high input power. This implied ethanol in high ethanol concentration droplets evaporated faster with low input power, but slower with high input power. To explain this: in low power condition, the large gradient of ethanol concentration between air/liquid interface led to a large driving force and violent internal flow which increased the evaporation of ethanol; while under a high power condition, air near the contact line was easily saturated because ethanol evaporated fast near base line in the very beginning with high input power, which impeded ethanol transport to air.

\section{ACKNOWLEDGEMENT}

This research is supported by the Research Grants Council (RGC) of the Government of the Hong Kong Special Administrative Region (HKSAR) with Project Nos. 618210 and 617812.

\section{REFERENCES}

[1] S. S. Abramchuk, A. R. Khokhlov, T. Iwataki, H. Oana and K. Yoshikawa, Direct observation of DNA molecules in a convection flow of a drying droplet, Europhysics Letters, Vol. 55, 2001, pp. 294-300

[2] M. Kaneda, K. Hyakuta, Yuu T., H. Ishizuka, and Jun F., Film Formation from Polymer Solution Using Inkjet Printing Method, Langmuir, Vol. 24, 2008, pp. 9102-9109

[3] V.M. Ha and C.L. Lai, Onset of Marangoni instability of a twocomponent evaporating droplet International Journal of Heat and Mass Transfer, Vol. 45, 2002, pp. 5143-5158

[4] S.S. Sazhin, A. Elwardany, P.A. Krutitskii, G. Castanet, F. Lemoine, E.M. Sazhina, M.R. Heikal, A simplified model for bicomponent droplet heating and evaporation, International
Journal of Heat and Mass Transfer, Vol. 53, 2010, pp. 44954505

[5] J. Armendariz and M. Matalon, Marangoni instability at a contaminated liquid-vapor interface of a burning thin film, Physics of Fluids, Vol15, 2003, pp. 1122

[6] D. K. Mandal and S. Bakshi, Evidence of oscillatory convection inside an evaporating multicomponent droplet in a closed chamber, Journal of Colloid and Interface Science, Vol. 378, 2012, pp.260-262

[7] I. Aharon and B. D. Shaw, Marangoni instability of bi-component droplet gasification, Physics of Fluids, Vol.8, 1996, pp.1820

[8] D. K. Mandal and S. Bakshi, Internal circulation in a single droplet evaporating in a closed chamber, International Journal of Multiphase Flow Journal, Vol.42 (2012), pp. 42-51

[9] Calvert, P., Inkjet printing for materials and devices, Chemistry of Materials, Vol.13 (2001), pp: 3299-3305

[10] Chang, S. T. and Velev, O. D., Evaporation-induced particle microseparations inside droplets floating on a chip, Langmuir, Vol.22 (2006), pp: $1459-1468$

[11] Picknett, R. G. and Bexon, R., The evaporation of sessile or pendant drops in still air, Journal of Colloid Interface Science, Vol.61 (1977), pp: 336-350

[12] Birdi K. S. and Vu D. T. J., Wettability and the evaporation rates of fluids from solid surfaces, Journal of Adhesion Science and Technology, Vol.7 (1993), pp: 485-493

[13] McHale, G., Rowan, S. M., Newton, M. I. and Banerjee, M. K., Evaporation and the wetting of a low-energy solid surface, The Journal of Physical Chemistry B, Vol.102(1998), pp: 1964-1967

[14] S. Dash and S. V. Garimella, Droplet evaporation dynamics on a superhydrophobic surface with negligible hysteresis, Langmuir, Vol.29 (2013), pp: 10785-10795

[15] Anantharaju, N., Panchagnula, M. and Neti, S., Evaporating drops on patterned surfaces: Transition from pinned to moving triple line, Journal of Colloid and Interface Science, Vol.337 (2009), pp: $176-182$

[16] F. R. Newbold and N. R. Amundson, A Model for Evaporation of a Multicomponent Droplet, AIChE Journal, Vol. 19(1973), pp: 22-30

[17] J. Tamim and W.L.H. Hallett, A continuous thermodynamics model for multi-component droplet vaporization, Chemical Engineering Science, Vol. 50 (1995), pp: 2933-2942

[18] Y. Zhao and H. H. Qiu, Measurements of multicomponent microdroplet evaporation by using Rainbow Refractometer and PDA, Experiments in Fluids, Vol.40 (2006), pp: 60-69

[19] K. H. Kang, S. J. Lee, C. M. Lee and I. S. Kang, Quantitative visualization of flow inside an evaporating droplet using the ray tracing method, Measurement Science and Technology, Vol.15 (2004), pp: 1104-1112 